\title{
Diagnosis of Ilioinguinal Nerve Injury Based on Electromyography and Ultrasonography: A Case Report
}

\author{
Hee-Mun Cho, MD, Dong-Sik Park, MD, Dong Hyun Kim, MD, Ho-Sung Nam, MD
}

Department of Rehabilitation Medicine, Hallym University Kangdong Sacred Heart Hospital, Hallym University College of Medicine, Seoul, Korea

\begin{abstract}
Being located in the hypogastric area, the ilioinguinal nerve, together with iliohypogastric nerve, can be damaged during lower abdominal surgeries. Conventionally, the diagnosis of ilioinguinal neuropathy relies on clinical assessments, and standardized diagnostic methods have not been established as of yet. We hereby report the case of young man who presented ilioinguinal neuralgia with symptoms of burning pain in the right groin and scrotum shortly after receiving inguinal herniorrhaphy. To raise the diagnostic certainty, we used a realtime ultrasonography (US) to guide a monopolar electromyography needle to the ilioinguinal nerve, and then performed a motor conduction study. A subsequent US-guided ilioinguinal nerve block resulted in complete resolution of the patient's neuralgic symptoms.
\end{abstract}

Keywords Ilioinguinal neuralgia, Electromyography, Ultrasonography

\section{INTRODUCTION}

The ilioinguinal nerve arises from the undivided Ll anterior primary ramus, and bears the typical characteristic features of the thoracic nerve, which distributes segmentally [1]. Increased risk to the ilioinguinal nerve injury is well-recognized in inguinal herniorrhaphy, open appendectomy, and major gynecologic surgeries [2]. In severe injury cases, it produces burning or radiating pain on the inguinal region and genitalia corresponding to the sen- sory distribution of the nerve. Up until now, the diagnosis of ilioinguinal nerve injury has been largely dependent upon clinical assessments: (1) previous surgical history, (2) pain and impaired sensory perception in the involved nerve distribution, and (3) pain relief after local infiltration of anesthetic in the area of the nerve.

To our knowledge, the standardized electrodiagnostic method of ilioinguinal nerve injury has not been established, and only a few case reports or pilot studies with small sample sizes have been published [2-4].

Received July 8, 2016; Accepted September 6, 2016

Corresponding author: Dong Hyun Kim

Department of Rehabilitation Medicine, Hallym University Kangdong Sacred Heart Hospital, Hallym University College of Medicine, 150 Seongan-ro, Gangdong-gu, Seoul 05355, Korea. Tel: +82-2-2225-2789, Fax: +82-2-473-8101, E-mail: skyler02@hallym.or.kr

ORCID: Hee-Mun Cho (http://orcid.org/0000-0003-3982-0703); Dong-Sik Park (http://orcid.org/0000-0002-8977-7099); Dong Hyun Kim (http://orcid. org/0000-0002-3102-385X); Ho-Sung Nam (http://orcid.org/0000-0003-3188-2053).

(c) This is an open-access article distributed under the terms of the Creative Commons Attribution Non-Commercial License (http://creativecommons.org/ licenses/by-nc/4.0) which permits unrestricted noncommercial use, distribution, and reproduction in any medium, provided the original work is properly cited. Copyright (c) 2017 by Korean Academy of Rehabilitation Medicine 
In this case, the authors present a series of diagnostic processes of a patient suspected with ilioinguinal nerve injury. Based upon the clinical presentations-history of inguinal herniorrhaphy and typical sensory abnormalities in the cutaneous distribution of the nerve-we performed ultrasonography (US) guided electromyography (EMG), US evaluation, and subsequent US-guided nerve block.

\section{CASE REPORT}

A-19-year-old man presented right groin pain after herniorrhaphy. His chief complaint was a stabbing pain which got worse by position change, such as hip joint extension, rotation or walking. He underwent herniorrhaphy on both sides sequentially due to bilateral inguinal hernias (Fig. 1). On both sides, the Lichtenstein technique was applied for surgical treatment. The first herniorrhaphy, on the left side, was completed successfully 1 year ago, without complications. However, 6 months later, and shortly after the herniorrhaphy on the right side was performed, complications arose. The patient presented burning pains in the right groin and scrotum. Upon physical examination, he demonstrated severe hypesthesia from the medial thigh to the femoral triangle, and in the abdominal wall overlying the symphysis of the pubic and right root of penis, as well as the upper part of the scrotum. No significant motor weakness in the trunk or low extremities was observed. For the electrodiagnosis, motor conduction study and needle EMG (Sierra wave; Cadwell, Kennewick, WA, USA) on the bilateral ilioinguinal nerve was performed with routine tibial, peroneal and sural conduction studies. Needle EMG on the right lower extremity and paraspinal muscles was also performed. Needle stimulation of the ilioinguinal nerve was conducted $3 \mathrm{~cm}$ medial and $3 \mathrm{~cm}$ inferior to the anterior superior iliac spine (ASIS) with a $50-\mathrm{mm}$, 26-gauge disposable pre-wired monopolar needle (Technomed Europe, Maastricht, the Netherlands). Compound muscle action potential (CMAP) was recorded as a surface recording at motor point over the lower abdomen ( $1 \mathrm{~cm}$ lateral to the midline and $1-2 \mathrm{~cm}$ above the pubic symphysis over the lower abdomen; most caudal segment of internal oblique abdominal muscle) by using a disposable silver chloride disk, $2 \mathrm{~cm}$ in diameter (Natus

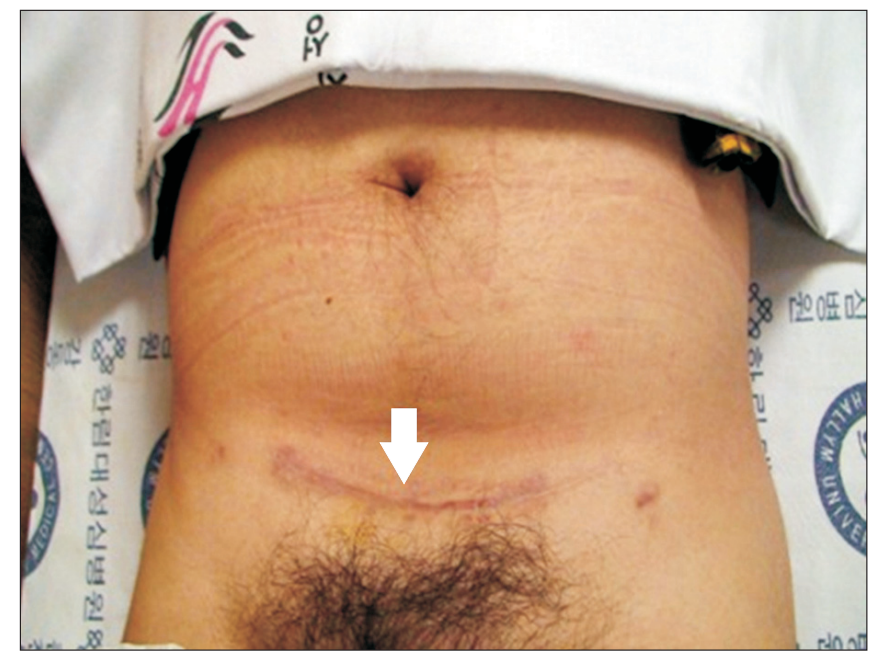

Fig. 1. Herniorrhaphy site of the patient (white arrow).
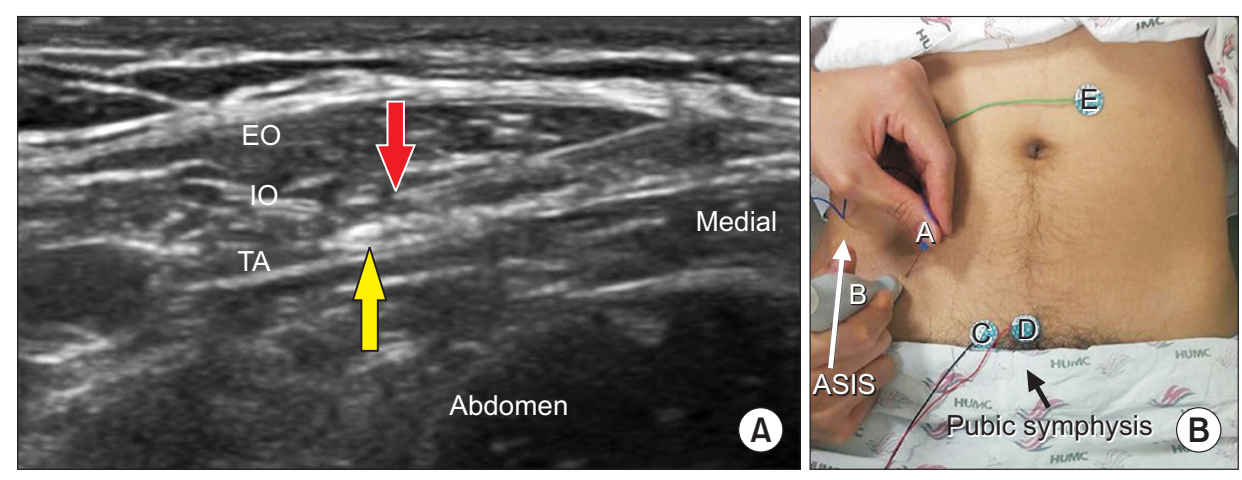

Fig. 2. Ilioinguinal motor conduction study. (A) Ultrasound image of the ilioinguinal nerve and monopolar needle between the internal abdominal oblique muscle and transverses abdominis muscle (red arrow, tip of monopolar needle; yellow arrow, ilioinguinal nerve; EO, external abdominal oblique muscle; IO, internal abdominal oblique muscle; TA, transversus abdominis muscle). (B) Demonstration of conduction study (A, needle electrode; B, probe; C, recording electrode; D, reference electrode; E, ground electrode). 
Neurology, Middleton, WI, USA).

In order to localize the ilioinguinal nerve, we located the linear US probe (L5-13IS; Samsung, Seoul, Korea) perpendicular to the inguinal ligament just medial to ASIS (Fig. 2B), and the needle tip was advanced gently towards the inferior and lateral direction under US-guidance (UGEO G60; Samsung), until it penetrated abdominal oblique muscles and nearly reached the ilioinguinal nerve (Fig. 2A). After placement of the needle tip, the patient's tingling sensations in the groin and scrotal area were monitored while applying an electrical stimulation of $10 \mathrm{~mA}$, stimulus duration of $200 \mathrm{~ms}$, at $1 \mathrm{~Hz}$.

A motor conduction study on the right ilioinguinal nerve revealed no response (Fig. 3B), while CMAP was obtained from the left side with a latency of $4.8 \mathrm{~ms}$, amplitude of $0.9 \mathrm{mV}$ (Fig. 3A). During EMG using a 50- $\mathrm{mm}, 26$-gauge disposable pre-wired monopolar needle, abnormal spontaneous activities were observed at the most caudal segment of the internal abdominal oblique muscle (Fig. 4). On US evaluation, diffuse fibrotic change was observed in the caudal segment of the right internal abdominal oblique and transversus abdominis muscle (Fig. 5B), while the left lower abdominal wall showed no change (Fig. 5A). The clinical symptoms and EMG study of the lower abdominal US suggested the diagnosis of right ilioinguinal nerve injury. Under strong suspicion of the diagnosis, ilioinguinal nerve block was performed.

An injectable monopolar needle electrode with a 50$\mathrm{mm}, 26$-gauge needle (Chalgren, London, UK) was inserted $3 \mathrm{~cm}$ inferior and $3 \mathrm{~cm}$ medial to the ASIS under US-guidance, with low voltage electrical stimulation to localize the ilioinguinal nerve. After precise targeting, 4
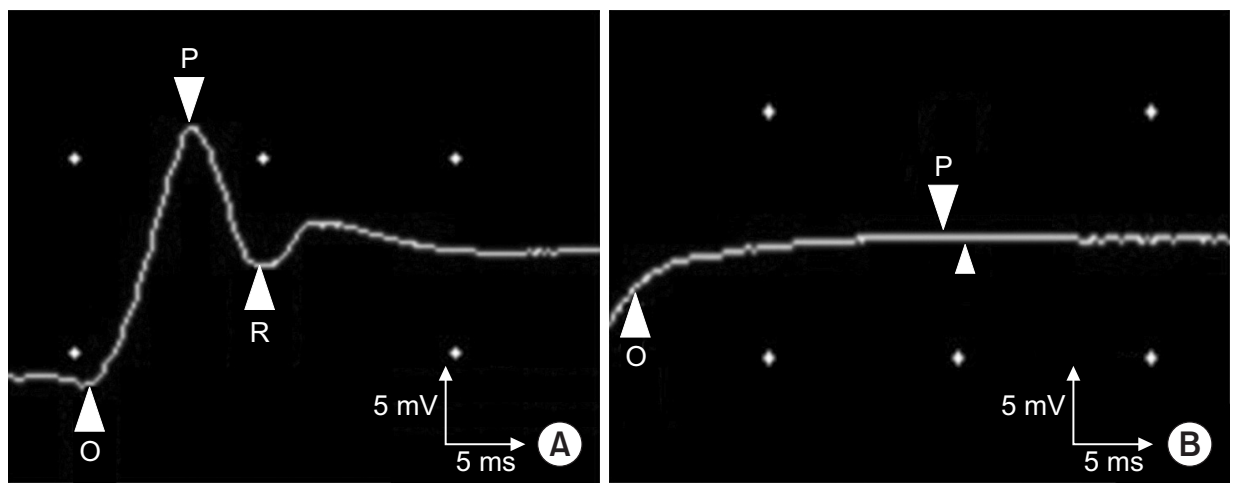

Fig. 3. Motor conduction study of ilioinguinal nerve. (A) Compound muscle action potential (CMAP) of left internal abdominal oblique muscle (latency, $4.8 \mathrm{~ms}$; amplitude, $0.9 \mathrm{mV}$ ). (B) CMAP of right internal abdominal oblique muscle (no response).

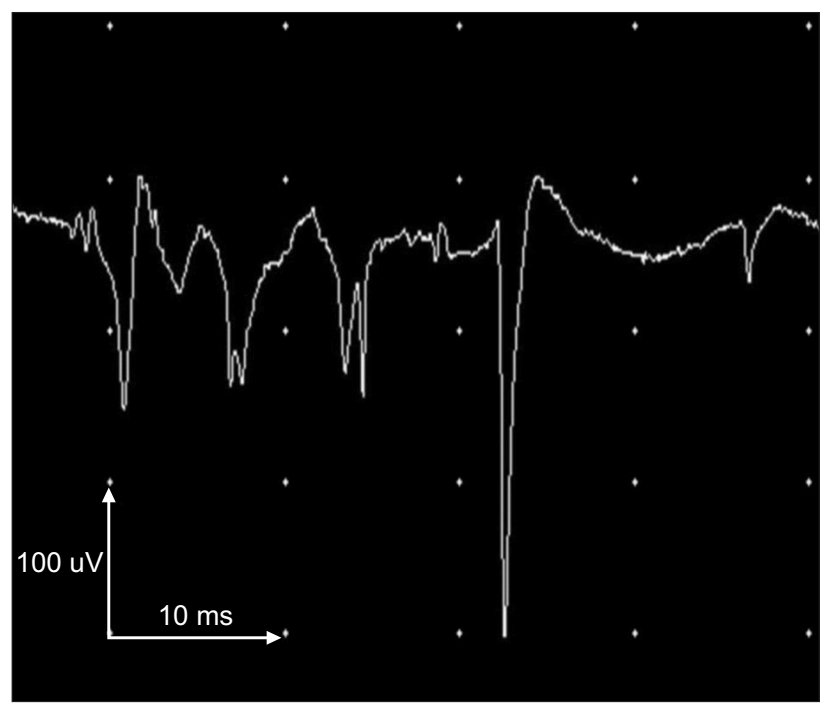

Fig. 4. Abnormal spontaneous activities in needle electromyography at the most caudal segment of the right internal abdominal oblique muscle.
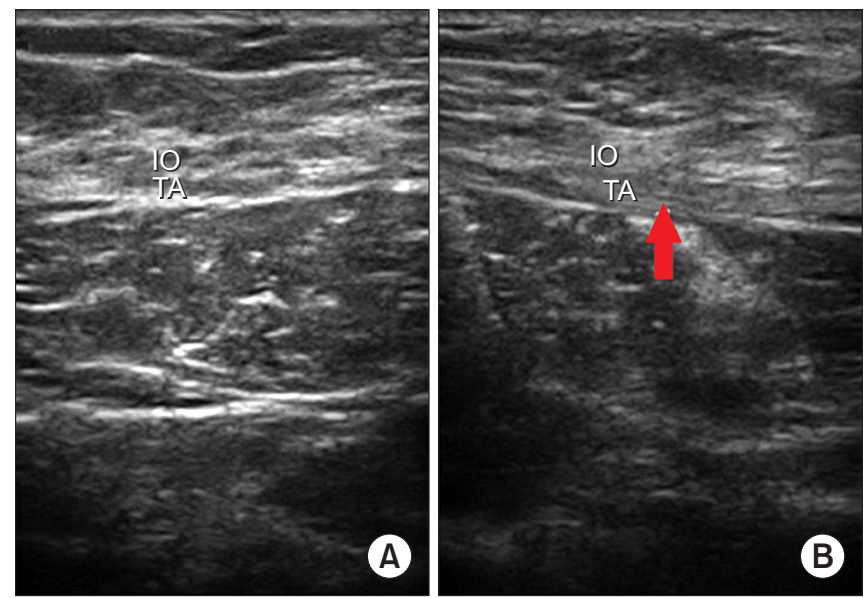

Fig. 5. Ultrasonography of lower abdominal muscle. (A) Left side (normal), (B) right side (red arrow, diffuse fibrotic change of muscles). IO, internal abdominal oblique muscle; TA, transversus abdominis muscle. 
$\mathrm{mL}$ of $2 \%$ lidocaine and $40 \mathrm{mg}$ of triamcinolone acetonide was injected. Soon after the injection, the groin and scrotum pain disappeared and symptom relief maintained for at least 60 days.

\section{DISCUSSION}

In order to increase the diagnostic accuracy of ilioinguinal nerve injury, motor conduction studies as well as needle EMG and US evaluations were performed. Although the electrodiagnosis of ilioinguinal nerve injury had been reported in previous studies [3,4], the possibility of mis-stimulating an iliohypogastric nerve or costimulating adjacent iliohypogastric nerves cannot be ruled out with the blind positioning of the EMG needle using anatomic landmarks. To avoid this pitfall, we used direct visualization of the monopolar EMG needle tip under real-time US guidance. A linear US probe was placed perpendicular to the inguinal ligament, and we monitored the accessibility of the needle tip near the ilioinguinal nerve located inferior to the iliohypogastric nerve.

Internal abdominal oblique muscles are supplied by segmental innervations of the lower intercostals, as well as the iliohypogastric and ilioinguinal nerves. Although studies of the anatomic landmarks dividing each segment are still inconclusive, the ilioinguinal nerve is known to innervate the most caudal segment of the internal abdominal oblique muscle inferior to the segment innervated by the iliohypogastric nerve $[5,6]$. Therefore, the recording of ilioinguinal CMAP was conducted by pubic symphysis [4].

Ellis et al. [3] had reported the mean ilioinguinal CMAP amplitude as $1.2 \mathrm{mV}$ (range, $0.3-5.0 \mathrm{mV}$ ) in a pilot study with 10 healthy candidates, and mentioned the positive side to side correlation of CMAP, rendering the unaffected side as a reliable amplitude standard. No articles have systematically reported the normal reference range of inguinal nerve CMAP since Ellis. In our case, the left ilioinguinal CMAP amplitude was recorded as $1.2 \mathrm{mV}$ while no response was elicited from the right side. Also, abnormal spontaneous activities were observed in the needle EMG of the right internal abdominal oblique muscles. Based on the patient's clinical symptoms and corresponding electrodiagnostic findings, we could make the diagnosis of ilioinguinal nerve injury, and the complete relief of symptoms after the subsequent nerve block confirmed the diagnosis.

We experienced a case of unilateral ilioinguinal nerve injury following herniorrhaphy, and report the series of diagnostic processes as well as the clinical outcomes after US-guided nerve block. Further research with a larger group of patients should follow to establish this diagnostic technique as well as to set up the normal reference range of ilioinguinal nerve CMAP.

\section{CONFLICT OF INTEREST}

No potential conflict of interest relevant to this article was reported.

\section{REFERENCES}

1. Rozen WM, Tran TM, Ashton MW, Barrington MJ, Ivanusic JJ, Taylor GI. Refining the course of the thoracolumbar nerves: a new understanding of the innervation of the anterior abdominal wall. Clin Anat 2008;21: 325-33.

2. ter Meulen BC, Peters EW, Wijsmuller A, Kropman RF, Mosch A, Tavy DL. Acute scrotal pain from idiopathic ilioinguinal neuropathy: diagnosis and treatment with EMG-guided nerve block. Clin Neurol Neurosurg 2007;109:535-7.

3. Ellis RJ, Geisse H, Holub BA, Swenson MR. Ilioinguinal nerve conduction. Muscle and Nerve 1992;10:1194.

4. Karakayali F, Karatas M, Ozcelik U, Ekici Y, Basaran $\mathrm{O}$, Moray G, et al. Influence of synthetic mesh on ilioinguinal nerve motor conduction and chronic groin pain after inguinal herniorrhaphy: a prospective randomized clinical study. Int Surg 2007;92:344-50.

5. Tagliafico A, Bignotti B, Cadoni A, Perez MM, Martinoli C. Anatomical study of the iliohypogastric, ilioinguinal, and genitofemoral nerves using highresolution ultrasound. Muscle Nerve 2015;51:42-8.

6. Whiteside JL, Barber MD, Walters MD, Falcone T. Anatomy of ilioinguinal and iliohypogastric nerves in relation to trocar placement and low transverse incisions. Am J Obstet Gynecol 2003;189:1574-8. 